\title{
Review of biodegradable synthetic-based drilling fluid: progression, performance and future prospect
}

\begin{abstract}
This paper provides a comprehensive review on ester based drilling fluid (EBDF). It is no secret that esters with biodegradability and bioaccumulation attributes are among the promising alternatives to synthetic base oil in drilling fluids. The findings from the literature explained the critical parameters for drilling fluid base which are i) kinematic viscosity, ii) pour point and iii) flash point iv) thermal stability and v) hydrolytic stability and vi) elastomer compatibility. In an ideal case, an EBDF requires base oil with low viscosity, low pour, high flash point, high thermal and hydrolytic stability and compatibility with existing elastomer. However in the real application, these requirements may not be the same as the bottom hole condition which is always subjected to high pressure and high temperature environment. At the moment, the performance of EBDF is considered outstanding for normal borehole depth and complexity. Nevertheless the constraints such as low temperature at the seabed while high temperature and high pressure at the bottom hole may be slightly different when dealing with an EBDF. This is due to its unique molecular structure of ester. Affected parameters include i) high kinematic viscosity, ii) hydrolytic degradation and iii) thermal stability. Failure in managing these parameters may lead to detrimental impacts on the drilling fluid performances and the fluid's stabilities. The application of low viscosity, high thermal and hydrolytic properties of esters and combination with unique carbon based nanomaterials into formulation might be able to close the gap of current EBDF performances.
\end{abstract}

Keyword: Ester based mud; Green energy; Drilling fluid; Nanomaterials; Esters performance; Rheological properties 